\title{
Rapid Method for the Determination of 180 Pesticide Residues in Foods by Gas Chromatography/Mass Spectrometry and Flame Photometric Detection
}

\author{
Masahiro OKIHASHI, * Yoko Kitagawa, Kazuhiko AkUtsu, Hirotaka OBana and Yukio Tanaka \\ Osaka Prefectural Institute of Public Health, Nakamichi 1-3-69, Higashinari-ku, Osaka 537-0025, Japan
}

(Received March 2, 2005; Accepted July 6, 2005)

\begin{abstract}
A method was established for the determination of 180 pesticide residues in fruits and vegetables. The procedure involved extraction with acetonitrile, followed by a salting-out step with anhydrous $\mathrm{MgSO}_{4}$ and $\mathrm{NaCl}$. Removal of sediment and water was performed simultaneously by centrifugation. Co-extractives were removed with a double-layered SPE column, and graphitized carbon black and primary secondary amine (GCB/PSA) solid phase extraction cleanup cartridge. The eluate was determined by GC/FPD and GC/MS without further cleanup. Recovery data were obtained by fortifying 9 matrices at $0.05-0.1 \mu \mathrm{g} / \mathrm{g}$. Recoveries of 180 pesticides were mainly $70-110 \%$ and the relative standard deviation (RSD) was below $25 \%$. Limits of detection ranged between 0.01 and $0.05 \mu \mathrm{g} / \mathrm{g}$ for tested pesticides. C Pesticide Science Society of Japan
\end{abstract}

Keywords: pesticide, residue analysis, multiresidue, graphitized carbon black / primary secondary amine.

\section{INTRODUCTION}

In Japan, maximum residue levels (MRLs) have been set for over 200 pesticides in the last decade, and this number will be increased to over 400 in 2006. In addition, agricultural products that contain pesticides not on MRL lists will be excluded from the market as illegal, with a positive-list system to be introduced in the fiscal year of 2006.

Pesticide residue analysis of foods has been performed by numerous governments and private laboratories throughout the world. ${ }^{1-3)}$ Regulatory agencies involved in the monitoring of pesticide residues in foods require fast and efficient multiresidue methods with a broad scope of application in order to maximize the coverage of their monitoring activities. Modern residue monitoring programs are expected to be responsive to the latest developments in agriculture and new legislation.

To date, many multiresidue analytic methods have been reported. $^{4-6)}$ Some of them require special instruments for extraction or cleanup. The system for supercritical fluid extraction (SFE), ${ }^{7,8)}$ accelerated solvent extraction (ASE), ${ }^{9-11)}$ and gel permeation chromatography $(\mathrm{GPC})^{12,13)}$ operate automatically. But only one sample is processed at a time, and the set-

\footnotetext{
* To whom correspondence should be addressed.

E-mail: okihasi@iph.pref.osaka.jp

(C) Pesticide Science Society of Japan
}

tings must be changed for each sample, consequently the cost is high. The aim of this study was to develop a simple and efficient multiresidue analysis that takes just one day and does not require expensive instruments for sample preparation. The main focus was to shorten the analytical process during extraction and cleanup. Anastassiades et al. reported a rapid approach to the analysis of pesticide residues in fruits and vegetables, named QuEChERS. ${ }^{14)}$ We examined QuEChERS and found that the method contained a respective point, small size liquid-liquid partitioning, and also found two negative aspects, weak extraction potency (shake) and insufficient cleanup (batch). In this study, we developed a more efficient method, adapting a cleanup cartridge using a graphitized carbon black (GCB) and primary secondary amine (PSA) double-layered (GCB/PSA) solid phase extraction (SPE) for the analysis of 180 pesticides in fruit and vegetable samples. These pesticides were detected by gas chromatography mass spectrometry (GC/MS) in the electronic ionization mode (EI) and negative chemical ionization mode (NCI), and by GC with a flame photometric detector (GC/FPD). The newly described method would compensate for the negative aspects of the QuEChERS method.

\section{MATERIALS AND METHODS}

1. Apparatus

1.1. Electron ionization (EI) mode GC/MS

A POLARIS Q ion trap mass spectrometer (Thermo Electron 
Corp., USA) equipped with a TRACE GC Ultra; column, Rtx-5 ms capillary column $30 \mathrm{~m} \times 0.25 \mathrm{~mm} \times 0.25 \mu \mathrm{m}$ (Restek Corp., USA); a helium carrier gas flow, $1.5 \mathrm{ml} / \mathrm{min}$; injection temperature, $250^{\circ} \mathrm{C}$; transfer line temperature, $280^{\circ} \mathrm{C}$; ion source temperature, $200^{\circ} \mathrm{C}$; ion mode, electronic ionization / scan mode; oven temperature program: $60^{\circ} \mathrm{C}$ for $1 \mathrm{~min}$, $8^{\circ} \mathrm{C} / \mathrm{min}$ to $280^{\circ} \mathrm{C}$ and held for $5 \mathrm{~min}$; splitless injection at a volume of $1 \mu \mathrm{l}$ by a AS-2000 auto sampler.

1.2. Negative chemical ionization (NCI) mode GC/MS A GCMS-QP2010 gas chromatograph / mass spectrometer (Shimadzu, Japan); column, DB-5 capillary column 30 $\mathrm{m} \times 0.25 \mathrm{~mm} \times 0.25 \mu \mathrm{m}(\mathrm{J} \& \mathrm{~W}$ Scientific, USA); helium carrier gas flow, $1.7 \mathrm{ml} / \mathrm{min}$; injection temperature, $250^{\circ} \mathrm{C}$; interface temperature, $250^{\circ} \mathrm{C}$; ion source temperature, $200^{\circ} \mathrm{C}$; ion mode, negative chemical ionization / selected ion monitoring mode; reaction gas, methane; oven temperature program: $60^{\circ} \mathrm{C}$ for $1 \mathrm{~min}, 20^{\circ} \mathrm{C} / \mathrm{min}$ to $170^{\circ} \mathrm{C}$, then $6^{\circ} \mathrm{C} / \mathrm{min}$ to $300^{\circ} \mathrm{C}$ and held for $7 \mathrm{~min}$; splitless injection at a volume of $1 \mu \mathrm{l}$ by a Shimadzu AOC-20i auto injector.

\section{3. $G C / F P D$}

A GC-17A (Shimadzu, Japan) equipped with a flame photometric detector (FPD); column, DB-1701 capillary column 30 $\mathrm{m} \times 0.32 \mathrm{~mm} \times 0.25 \mu \mathrm{m}$ ( $\mathrm{J} \& \mathrm{~W}$ Scientific, USA); helium carrier gas flow, $2.0 \mathrm{ml} / \mathrm{min}$; injection temperature, $250^{\circ} \mathrm{C}$; detector temperature, $280^{\circ} \mathrm{C}$; oven temperature program: $80^{\circ} \mathrm{C}$ for $2 \mathrm{~min}, 20^{\circ} \mathrm{C} / \mathrm{min}$ to $180^{\circ} \mathrm{C}$, then $4^{\circ} \mathrm{C} / \mathrm{min}$ to $260^{\circ} \mathrm{C}$, then $10^{\circ} \mathrm{C} / \mathrm{min}$ to $280^{\circ} \mathrm{C}$ for $5 \mathrm{~min}$, splitless injection at a volume of $2 \mu \mathrm{l}$ by a Shimadzu AOC-14 auto injector.

\section{Chemicals}

Acetonitrile, toluene, acetone and $n$-hexane were of pesticide analysis grade from Wako Pure Chemical Ind. (Japan). Anhydrous magnesium sulfate, sodium chloride and acetic acid were of analytical grade from Wako. SPE tubes, GCB; Supelclean ENVI-Carb (250 mg) and GCB/PSA; Supelclean ENVICarb/PSA $(500 \mathrm{mg} / 500 \mathrm{mg})$, were purchased from Supelco (USA). GCB/PSA SPE was preconditioned with a $30 \mathrm{ml} \mathrm{mix-}$ ture of acetonitrile-toluene ( $3: 1)$ containing $0.5 \%$ acetic acid.

Pesticide standards were obtained from Wako, Kanto Kagaku (Japan), Riedel de Haën (Germany), Hayashi Pure Chemical (Japan) and Dr. Ehrenstorfer G.m.b.H. (Germany). Each compound was dissolved in acetone to make a $1000 \mu \mathrm{g} / \mathrm{ml}$ stock standard solution. Mixed-compound intermediate solutions were prepared from stock solutions at concentrations ranging from 40 to $100 \mu \mathrm{g} / \mathrm{ml}$. Spiking solutions were prepared from intermediate solutions containing approximately 100 or 200 compounds at concentrations of $5 \mu \mathrm{g} / \mathrm{ml}$. Spiking solutions were used for fortifying the samples and also for the calculation after appropriate dilution.

\section{Sample Preparation}

All crops were purchased at a local market in Osaka and we confirmed that the concentrations of pesticide residues in foods were below detectable levels with the proposed method.

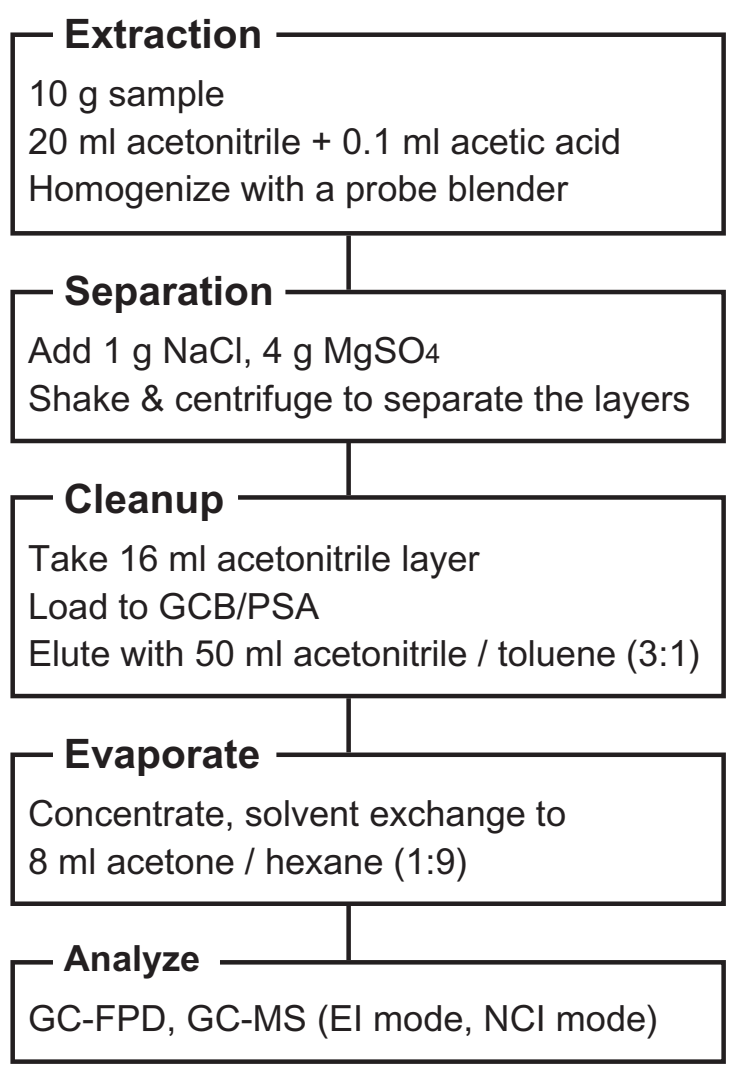

Fig. 1. Flow chart of the multiresidue method.

About 500-1000g of food was chopped in a QS-7 food processor (Toshiba, Japan) for more than 1 min to obtain thoroughly mixed homogenates.

An aliquot of $10 \mathrm{~g}$ of sample homogenate was weighed into a BLUE MAX $50 \mathrm{ml}$ polypropylene conical tube (Becton Dickinson, USA) and $100-200 \mu \mathrm{l}$ of spiking solution (5-10 $\mathrm{ng} / \mu \mathrm{l}$ for all compounds) was added. The mixture was left to stand for more than $30 \mathrm{~min}$ before extraction. The spiked sample was extracted with a mixture of $20 \mathrm{ml}$ of acetonitrile and $0.1 \mathrm{ml}$ of acetic acid by a HG30 homogenizer (Hitachi, Japan) for $1 \mathrm{~min}$. One gram of $\mathrm{NaCl}$ and $4 \mathrm{~g}$ of anhydrous $\mathrm{MgSO}_{4}$ were further added and shaken immediately for about $30 \mathrm{~s}$ with the screw cap on. ${ }^{14)}$ The extract was centrifuged for $10 \mathrm{~min}$ at $6000 \mathrm{rpm}$ using a Himac SCR 20B (Hitachi, Japan) to separate the sediment and water from the acetonitrile. Next, $16 \mathrm{ml}$ (equivalent to $8 \mathrm{~g}$ of sample) of the acetonitrile layer obtained after salting out was loaded into a GCB/PSA SPE tube. Pesticides were eluted with $50 \mathrm{ml}$ of acetonitrile-toluene $(3: 1)$. The eluate was evaporated and the residue was dissolved in $8 \mathrm{ml}$ of acetone-hexane $(1: 9)$ for GC/FPD and GC/MS analysis. The concentration of the sample represented by the test solution was $1 \mathrm{~g} / \mathrm{ml}$. Figure 1 summarizes the procedure.

Calibration was achieved by preparing matrix matched calibration standards from the extracts of blank samples in order to compensate for the matrix effect. Analytes were quantified 
by using a 3-point calibration with those matrix matched calibration standards corresponding to the spiked concentration.

\section{RESULTS AND DISCUSSION}

\section{Method Development}

Anastassiades et al. reported a quick, easy, cheap, effective, rugged and safe method named QuEChERS. ${ }^{14)}$ Pesticides were extracted by acetonitrile using a vortex mixer, the cleanup procedure was performed by dispersive-SPE using PSA particles, and the final extract was injected directly into the GC/MS system. They avoided the solvent evaporation and reconstitution steps to save time and labor. But we found that this procedure was not sufficient in removing food colorings such as chlorophyll, carotene, and water soluble materials, such as sugars and sodium chloride, when we used the QuEChERS method. We have introduced the positive aspects of QuEChERS, such as a small extraction scale, and phase separation with $\mathrm{MgSO}_{4}$ and $\mathrm{NaCl}$, to a conventional acetonitrile extraction and further improved the cleanup step. Duplicated analyses were performed for the comparison of extraction between the QuEChERS method (shaking) and our proposed method (homogenizing) using samples containing incurred pesticide residues. As shown in Table 1, the five detected pesticides, especially organochlorine pesticides, had lower values after shaking with QuEChERS than after vigorous mechanical homogenization with the newly proposed method.

Anastassiades et al. and Schenck et al. reported that the results with the vortexing procedure were similar to those with the blender for incurred pesticides. ${ }^{14,15)}$ It was suspected that the results might be affected by the difference in pesticides detected and the capability of our food processor. In any case, the probe homogenizer has an advantage over a shaker to break down foods into particles. The homogenizer needed a certain volume of solvent. Extraction was conducted with $10 \mathrm{~g}$ sample $+20 \mathrm{ml}$ acetonitrile. The homogenized extracts, $1: 2$ /sample: solvent ratio, were a darker color than the shaken extracts, $1: 1 /$ sample : solvent ratio. The conclusion was reached that homogenizing was superior to shaking as an extraction method. Moreover, we obtained broad peaks of weak intensity on GC/MS and GC/FPD chromatograms with direct injection of the acetonitrile solution.

We chose traditional SPE involving evaporation and reconstitution for removing hexane-insoluble sugars and salts. We did not use internal standards because triphenylphosphate, which is used in the QuEChERS method, was trapped in GCB. The separated acetonitrile contained a small amount of water. $^{16)}$ We disregarded the change in volume because we considered it to have little effect.

\section{Measurement}

Almost all of the targeted pesticides were measured by EI mode GC/MS, but food matrices were frequently detected and sometimes interfered with the results. To aid with identifica-
Table 1. Comparison of analytical results obtained using two extraction methods

\begin{tabular}{llll}
\hline \multicolumn{1}{c}{ Food } & \multicolumn{1}{c}{ Pesticide } & Shaking & Homogenizing \\
\hline & & & \multicolumn{1}{c}{$(\mathrm{ppm})$} \\
Pumpkin & Dieldrin & 0.011 & 0.013 \\
Pumpkin & Endrin & 0.009 & 0.012 \\
String bean & Dicofol & 0.14 & 0.34 \\
String bean & $o, p^{\prime}$-DDT & 0.011 & 0.025 \\
String bean & Methamidophos & 0.83 & 0.93 \\
\hline
\end{tabular}

tion, GC/FPD for organophosphorous pesticides and NCI mode GC/MS for organochlorin and pyrethroid pesticides were adopted. Organophosphorous pesticides were detected using GC/FPD with a DB-1701, a mid-polarity phase column. Acephate and methamidophos were little detected using GC/MS with a DB-5, a low polarity phase column. Azinphosmethyl and monocrotophos were not detected by GC/MS under the proposed conditions. Organochlorine and pyrethroid pesticides were detected with NCI mode GC/MS, which could detect halogenated compounds with high sensitivity. ${ }^{17)}$ Serious interference was not observed with NCI mode GC/MS and pyrethroids could be detected at lower levels than with GC/MS in the EI mode or GC with an electron capture detector (Fig. 2). Matrix enhancement effects were sometimes observed especially in EI mode GC/MS chromatograms. About $60 \%$ of pesticides showed unacceptably high responses $(>120 \%)$ with an orange matrix. Pesticide concentrations calculated with standards in solvent alone may be much higher than expected. Calibration was achieved by preparing matrix matched calibration standards from the extracts of blank samples, in order to compensate for the matrix effect.

\section{Cleanup}

GCB with $30 \mathrm{ml}$ of acetonitrile-toluene (3:1) was compared with GCB/PSA. The GCB column was effective at eliminating pigment and a primary secondary amine column could remove polar matrices and fatty acids. Extraction tests using both columns were conducted 5 times for each sample of lettuce, orange, and paprika. All extracts became clear after SPE, but the eluate from GCB contained some sediment and was dark. Next, $6 \mathrm{ml}$ of extract (equivalent to $6 \mathrm{~g}$ of sample) was dried in a preweighed test tube, and the amount of coextracted material was determined from the difference in weight after the extract had dried. Figure 3 shows that the double-layered SPE column showed about $40 \%$ or more cleanup for the residual weight of dried matrices in all samples, compared with single GCB. Figure 4 shows the total ion chromatogram of banana extracts eluted from GCB and GCB/PSA.

These chromatograms indicated that PSA reduced GC-detectable matrices. Saito et al. also reported that the combination of GCB and PSA provided excellent cleanup for removal 

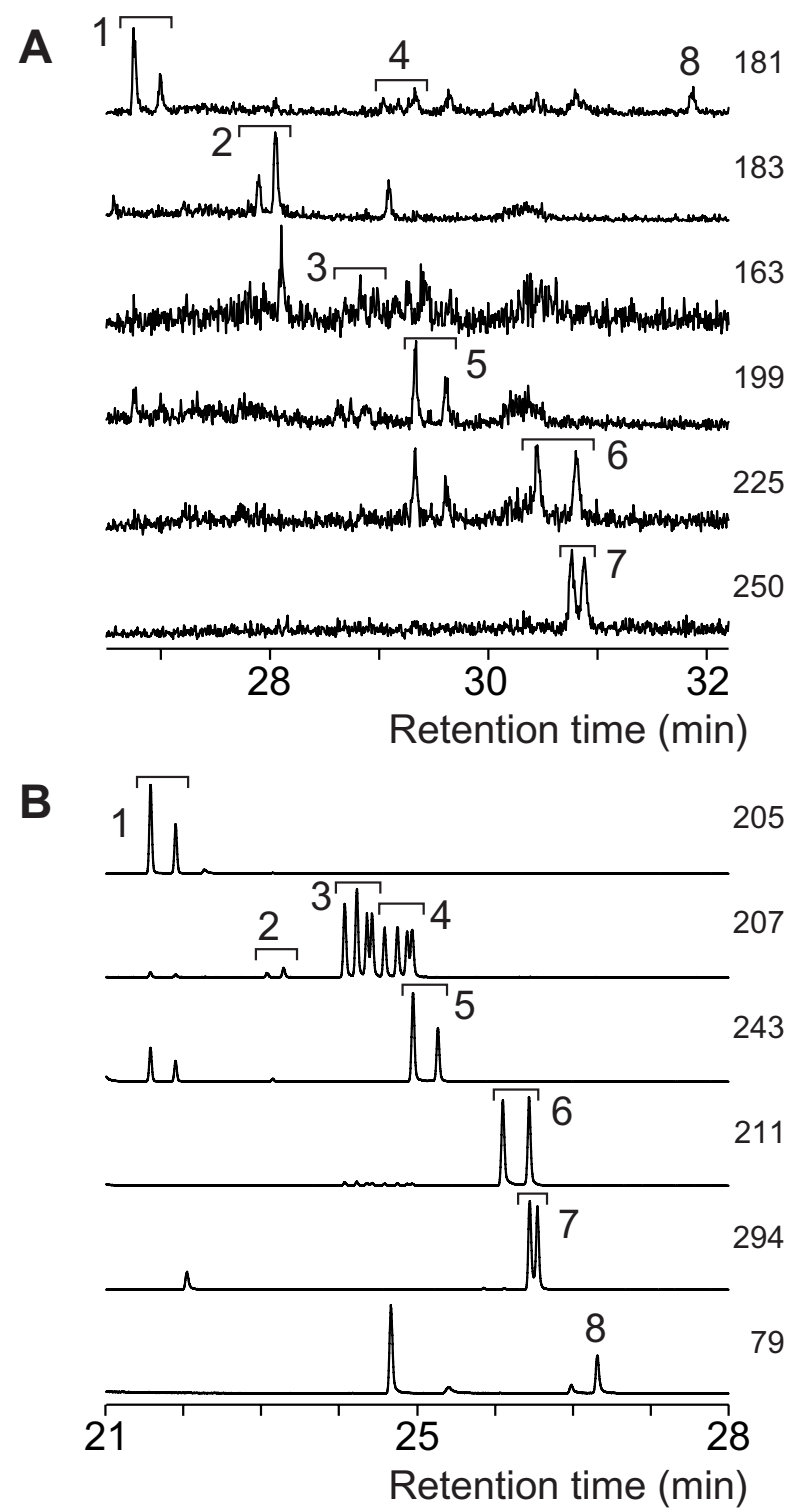

Fig. 2. GC/MS chromatograms of pyrethroid pesticides at $0.2 \mu \mathrm{g} / \mathrm{ml}$. A: EI mode (scan); B: NCI mode (SIM). 1: Cyhalothrin, 2: Permethrin, 3: Cyfluthrin, 4: Cypermethrin, 5: Flucythrinate, 6: Fenvalerate, 7: Fluvalinate, 8: Deltamethrin.

of matrix materials. ${ }^{18)}$ Almost all of the targeted pesticides were recovered sufficiently from GCB/PSA with the proposed procedure except chinomethionate and chlorothalonil. They were not recovered from GCB/PSA with acetonitrile-toluene ( $3: 1)$, though both pesticides were well recovered from GCB. The potency of the PSA column was examined using a mixedpesticide solution and the results showed that these pesticides were captured by PSA. PSA was capable of removing of fatty acids, and these pesticides might be captured as a result. We tried to weaken the effect of PSA by adding ethyl acetate or acetic acid to the mixture of acetonitrile-toluene. We found that the addition of $0.5 \%$ acetic acid improved the recovery of both pesticides from the double-layered SPE column. The re-

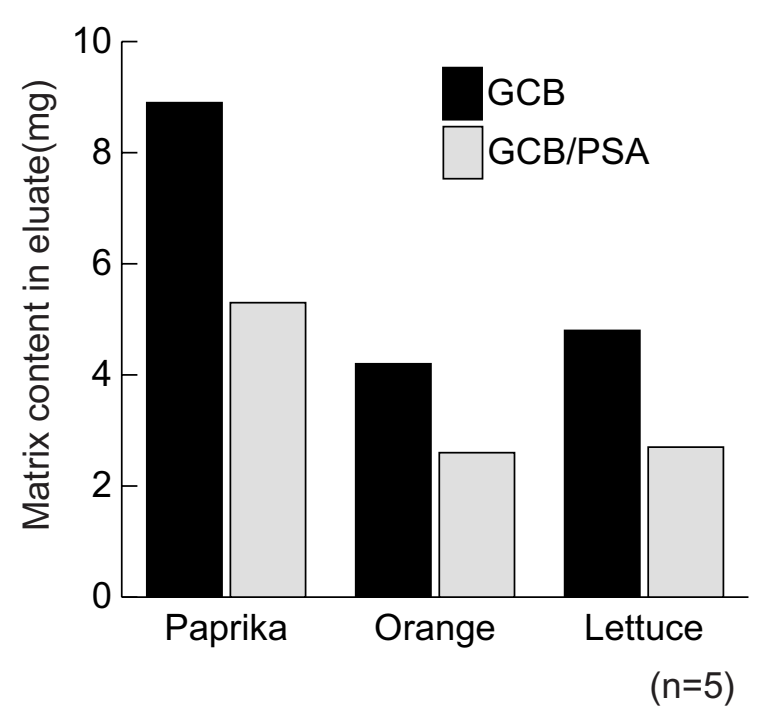

Fig. 3. Comparison of residual matrices in eluates obtained from two mini columns.

covery of chlorothalonil was over $70 \%$, but the recovery of chinomethionate was still below $50 \%$.

\section{Recovery Test}

The recovery tests were conducted 5 times for each sample of tomato, lettuce $(n=5 \times 2)$, orange and paprika at a level of $0.05 \mu \mathrm{g} / \mathrm{g}$, and apple, banana, broccoli, spinach and grapefruit at a level of $0.1 \mu \mathrm{g} / \mathrm{g}$. The data are summarized in Table 2 .

Recoveries of 180 pesticides were between 70 and $110 \%$ and the relative standard deviation (RSD) was below $25 \%$ at each spiked level except for some pesticides in spinach and broccoli. Organophosphorous pesticides had lower RSDs than other pesticides. It was speculated that GC/FPD was more accurate than GC/MS. In routine analysis, it is easy to recognize the negative results for organophosphorous pesticides from one copy of a flat chromatogram. The data from GC/MS is composed of many mass chromatograms and takes some time to confirm. GC/FPD is useful to shorten the time needed for

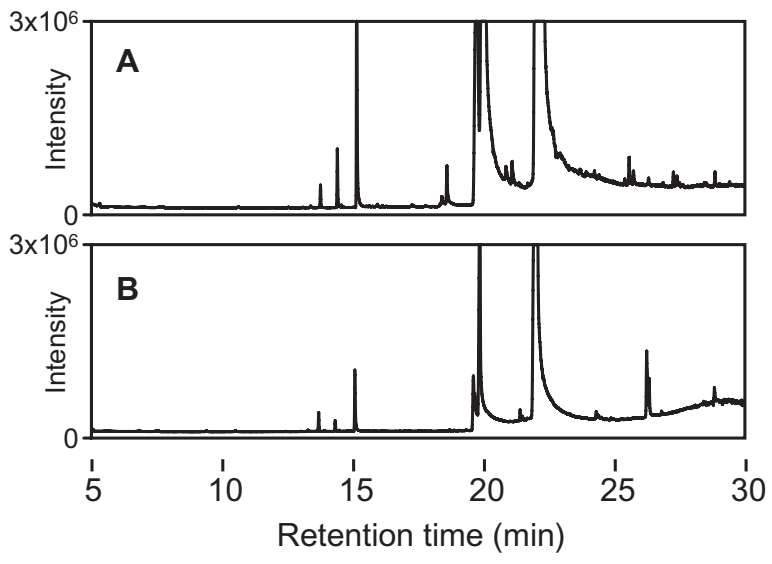

Fig. 4. Comparison of total ion chromatogram of banana extracts. A: GCB; B: GCB/PSA. 
Table 2. Recovery data for compounds determined by the multiresidue method

\begin{tabular}{|c|c|c|c|c|c|c|c|c|c|}
\hline Spiking level $(\mu \mathrm{g} / \mathrm{g})$ & & & & & 0.05 & & & 0.1 & \\
\hline Compound & Detector & $\begin{array}{c}\text { Monitor ion } \\
(\mathrm{m} / \mathrm{z})\end{array}$ & $\begin{array}{l}\text { LOD } \\
(\mu \mathrm{g} / \mathrm{g})\end{array}$ & $\mathrm{n}$ & $\begin{array}{c}\text { Mean } \\
\text { recovery }(\%)\end{array}$ & $\begin{array}{l}\text { RSD } \\
(\%)\end{array}$ & $\mathrm{n}$ & $\begin{array}{c}\text { Mean } \\
\text { recovery }(\%)\end{array}$ & $\begin{array}{l}\text { RSD } \\
(\%)\end{array}$ \\
\hline$\alpha$-BHC & $\mathrm{NCI}$ & 71 & 0.01 & $25^{a)}$ & 91 & 8 & $25^{d)}$ & 85 & 10 \\
\hline$\beta$-BHC & $\mathrm{NCI}$ & 71 & 0.01 & $25^{a)}$ & 93 & 9 & $25^{d)}$ & 86 & 8 \\
\hline$\gamma$-BHC & $\mathrm{NCI}$ & 71 & 0.01 & $25^{a)}$ & 93 & 8 & $25^{d)}$ & 86 & 8 \\
\hline$\delta$-BHC & $\mathrm{NCI}$ & 71 & 0.01 & $25^{a)}$ & 93 & 8 & $25^{d)}$ & 85 & 12 \\
\hline Tefluthrin & $\mathrm{NCI}$ & 241 & 0.01 & $25^{a)}$ & 93 & 11 & $25^{d)}$ & 87 & 9 \\
\hline Chlorothalonil & $\mathrm{NCI}$ & 266 & 0.01 & $25^{a)}$ & 73 & 30 & $25^{d)}$ & 72 & 22 \\
\hline Heptachlor & $\mathrm{NCI}$ & 300 & 0.01 & $25^{a)}$ & 100 & 11 & $25^{d)}$ & 85 & 7 \\
\hline Aldrin & $\mathrm{NCI}$ & 237 & 0.02 & $25^{a)}$ & 91 & 9 & $25^{d)}$ & 83 & 6 \\
\hline Dicofol & $\mathrm{NCI}$ & 250 & 0.01 & $25^{a)}$ & 91 & 12 & $25^{d)}$ & 90 & 16 \\
\hline Heptachlor-epoxide & $\mathrm{NCI}$ & 282 & 0.01 & $10^{b)}$ & 86 & 6 & $10^{e)}$ & 84 & 6 \\
\hline Captan & $\mathrm{NCI}$ & 150 & 0.02 & $23^{a)}$ & 87 & 19 & $10^{e)}$ & ND & - \\
\hline Procymidone & $\mathrm{NCI}$ & 282 & 0.02 & $25^{a)}$ & 93 & 11 & $25^{d)}$ & 86 & 9 \\
\hline$p p^{\prime}-\mathrm{DDE}$ & $\mathrm{NCI}$ & 35 & 0.01 & $25^{a)}$ & 91 & 10 & $25^{d)}$ & 84 & 9 \\
\hline Dieldrin & $\mathrm{NCI}$ & 237 & 0.01 & $25^{a)}$ & 92 & 11 & $25^{d)}$ & 87 & 9 \\
\hline Endrin & $\mathrm{NCI}$ & 237 & 0.01 & $25^{a)}$ & 96 & 11 & $25^{d)}$ & 87 & 12 \\
\hline Chlorobenzilate & $\mathrm{NCI}$ & 278 & 0.01 & $10^{b)}$ & 95 & 6 & $25^{d)}$ & 81 & 23 \\
\hline$p p^{\prime}-\mathrm{DDD}$ & $\mathrm{NCI}$ & 71 & 0.01 & $25^{a)}$ & 96 & 10 & $25^{d)}$ & 85 & 7 \\
\hline$o p^{\prime}$-DDT & $\mathrm{NCI}$ & 71 & 0.01 & $25^{a)}$ & 99 & 10 & $25^{d)}$ & 85 & 9 \\
\hline$p p^{\prime}$-DDT & $\mathrm{NCI}$ & 71 & 0.01 & $25^{a)}$ & 100 & 11 & $25^{d)}$ & 84 & 11 \\
\hline Captafol & $\mathrm{NCI}$ & 150 & 0.01 & $23^{a)}$ & 73 & 27 & $5^{f)}$ & 43 & 20 \\
\hline Cyhalothrin & $\mathrm{NCI}$ & 205 & 0.01 & $25^{a)}$ & 100 & 12 & $25^{d)}$ & 88 & 15 \\
\hline Permethrin & $\mathrm{NCI}$ & 207 & 0.02 & $25^{a)}$ & 98 & 14 & $25^{d)}$ & 87 & 19 \\
\hline Cyfluthrin & $\mathrm{NCI}$ & 207 & 0.01 & $25^{a)}$ & 101 & 14 & $25^{d)}$ & 87 & 12 \\
\hline Cypermethrin & $\mathrm{NCI}$ & 207 & 0.01 & $25^{a)}$ & 101 & 14 & $25^{d)}$ & 89 & 12 \\
\hline Flucythrinate & $\mathrm{NCI}$ & 243 & 0.01 & $25^{a)}$ & 101 & 14 & $25^{d)}$ & 82 & 18 \\
\hline Fenvalerate & $\mathrm{NCI}$ & 211 & 0.01 & $25^{a)}$ & 99 & 14 & $25^{d)}$ & 83 & 13 \\
\hline Fluvalinate & $\mathrm{NCI}$ & 294 & 0.01 & $25^{a)}$ & 105 & 13 & $25^{d)}$ & 82 & 17 \\
\hline Deltamethrin & $\mathrm{NCI}$ & 79 & 0.01 & $25^{a)}$ & 97 & 16 & $10^{e)}$ & 78 & 12 \\
\hline Dichlorvos & FPD & - & 0.01 & $25^{a)}$ & 89 & 7 & $25^{d)}$ & 85 & 6 \\
\hline Methamidophos & FPD & - & 0.01 & $25^{a)}$ & 71 & 14 & $25^{d)}$ & 62 & 24 \\
\hline Acephate & FPD & $(136)^{*}$ & 0.01 & $10^{b)}$ & 80 & 6 & $22^{d)}$ & 59 & 34 \\
\hline Ethoprophos & FPD & $(158)^{*}$ & 0.01 & $25^{a)}$ & 99 & 12 & $25^{d)}$ & 92 & 8 \\
\hline Dioxabenzofos & FPD & $(216)^{*}$ & 0.01 & $25^{a)}$ & 93 & 7 & $25^{d)}$ & 90 & 4 \\
\hline Terbufos & FPD & $(231)^{*}$ & 0.01 & $25^{a)}$ & 92 & 6 & $25^{d)}$ & 87 & 8 \\
\hline Diazinon & FPD & $(179)^{*}$ & 0.01 & $25^{a)}$ & 95 & 6 & $25^{d)}$ & 90 & 5 \\
\hline Iprobenfos & FPD & $(204)^{*}$ & 0.01 & $25^{a)}$ & 94 & 12 & $25^{d)}$ & 91 & 12 \\
\hline Dichlofenthion & FPD & $(279)^{*}$ & 0.01 & $25^{a)}$ & 96 & 7 & $25^{d)}$ & 88 & 6 \\
\hline Isazophos & FPD & $(172)^{*}$ & 0.01 & $10^{b)}$ & 85 & 12 & $10^{g)}$ & 85 & 10 \\
\hline Monocrotophos & FPD & - & 0.01 & $10^{b)}$ & 89 & 5 & $10^{e)}$ & 84 & 4 \\
\hline Cyanophos & FPD & $(243)^{*}$ & 0.01 & $10^{b)}$ & 84 & 9 & $10^{g)}$ & 87 & 6 \\
\hline Dimethoate & FPD & $(87)^{*}$ & 0.01 & $10^{b)}$ & 93 & 16 & $25^{d)}$ & 77 & 29 \\
\hline
\end{tabular}


Table 2. (Continued)

\begin{tabular}{|c|c|c|c|c|c|c|c|c|c|}
\hline Spiking level $(\mu \mathrm{g} / \mathrm{g})$ & & & & & 0.05 & & & 0.1 & \\
\hline Compound & Detector & $\begin{array}{l}\text { Monitor ion } \\
\qquad(m / z)\end{array}$ & $\begin{array}{l}\text { LOD } \\
(\mu \mathrm{g} / \mathrm{g})\end{array}$ & $\mathrm{n}$ & $\begin{array}{c}\text { Mean } \\
\text { recovery }(\%)\end{array}$ & $\begin{array}{l}\text { RSD } \\
(\%)\end{array}$ & $\mathrm{n}$ & $\begin{array}{c}\text { Mean } \\
\text { recovery }(\%)\end{array}$ & $\begin{array}{l}\text { RSD } \\
(\%)\end{array}$ \\
\hline Chlorpyrifos-methyl & FPD & $(286)^{*}$ & 0.01 & $10^{b)}$ & 86 & 8 & $25^{d)}$ & 85 & 7 \\
\hline Tolclofos-methyl & FPD & $(265)^{*}$ & 0.01 & $25^{a)}$ & 94 & 6 & $25^{d)}$ & 91 & 4 \\
\hline Pirimiphos-methyl & FPD & $(290)^{*}$ & 0.01 & $25^{a)}$ & 95 & 6 & $25^{d)}$ & 92 & 4 \\
\hline Chlorpyrifos & FPD & $(258)^{*}$ & 0.01 & $25^{a)}$ & 95 & 6 & $25^{d)}$ & 89 & 6 \\
\hline Phosphamidon & FPD & $(127)^{*}$ & 0.02 & $10^{b)}$ & 91 & 3 & $10^{e)}$ & 89 & 4 \\
\hline Fenthion & FPD & $(278)^{*}$ & 0.01 & $25^{a)}$ & 91 & 9 & $25^{d)}$ & 86 & 7 \\
\hline Malathion & FPD & $(127)^{*}$ & 0.01 & $25^{a)}$ & 93 & 7 & $24^{(d)}$ & 87 & 17 \\
\hline Bromophos-methyl & FPD & $(331)^{*}$ & 0.01 & $10^{b)}$ & 86 & 8 & $10^{g)}$ & 84 & 9 \\
\hline Fenitrothion & FPD & $(260)^{*}$ & 0.01 & $25^{a)}$ & 94 & 7 & $24^{d)}$ & 86 & 11 \\
\hline Dimethylvinphos & FPD & $(295)^{*}$ & 0.01 & $10^{b)}$ & 90 & 3 & $10^{e)}$ & 88 & 5 \\
\hline Isofenphos & FPD & $(213)^{*}$ & 0.01 & $25^{a)}$ & 96 & 7 & $25^{d)}$ & 93 & 4 \\
\hline Phenthoate & FPD & $(274)^{*}$ & 0.01 & $25^{a)}$ & 95 & 7 & $25^{d)}$ & 92 & 4 \\
\hline Fosthiazate & FPD & $(227)^{*}$ & 0.02 & $10^{b)}$ & 91 & 3 & $10^{e)}$ & 84 & 11 \\
\hline Prothiofos & FPD & $(309)^{*}$ & 0.01 & $25^{a)}$ & 95 & 6 & $25^{d)}$ & 91 & 6 \\
\hline Tetrachlorvinphos & FPD & $(329)^{*}$ & 0.01 & $10^{b)}$ & 90 & 4 & $10^{e)}$ & 82 & 12 \\
\hline Methidathion & FPD & $(145)^{*}$ & 0.01 & $25^{a)}$ & 94 & 7 & $25^{d)}$ & 94 & 10 \\
\hline Profenofos & FPD & $(337)^{*}$ & 0.01 & $10^{b)}$ & 92 & 4 & $10^{e)}$ & 88 & 6 \\
\hline Butamifos & FPD & $(286)^{*}$ & 0.01 & $25^{a)}$ & 96 & 7 & $25^{d)}$ & 95 & 5 \\
\hline Fenamiphos & FPD & $(303)^{*}$ & 0.01 & $10^{b)}$ & 90 & 4 & $10^{e)}$ & 89 & 2 \\
\hline Isoxathion & FPD & $(177)^{*}$ & 0.01 & $25^{a)}$ & 96 & 8 & $25^{d)}$ & 93 & 6 \\
\hline Ethion & FPD & $(231)^{*}$ & 0.01 & $25^{a)}$ & 97 & 7 & $25^{d)}$ & 93 & 5 \\
\hline Edifenphos & FPD & $(310)^{*}$ & 0.02 & $10^{b)}$ & 87 & 6 & $25^{d)}$ & 76 & 22 \\
\hline Triazophos & FPD & $(162)^{*}$ & 0.01 & $10^{b)}$ & 95 & 4 & $10^{e)}$ & 95 & 3 \\
\hline Cyanofenphos & FPD & $(169)^{*}$ & 0.01 & $10^{b)}$ & 92 & 3 & $10^{e)}$ & 92 & 3 \\
\hline EPN & FPD & $(169)^{*}$ & 0.01 & $25^{a)}$ & 96 & 9 & $25^{d)}$ & 91 & 5 \\
\hline Piperophos & FPD & $(320)^{*}$ & 0.01 & $10^{b)}$ & 92 & 6 & $10^{e)}$ & 93 & 5 \\
\hline Pyridaphenthion & FPD & $(340)^{*}$ & 0.01 & $25^{a)}$ & 93 & 9 & $25^{d)}$ & 91 & 10 \\
\hline Phosalone & FPD & $(182)^{*}$ & 0.02 & $25^{a)}$ & 95 & 8 & $25^{d)}$ & 93 & 8 \\
\hline Azinphos-methyl & FPD & - & 0.02 & $10^{b)}$ & 87 & 5 & $10^{e)}$ & 84 & 12 \\
\hline Pyraclofos & FPD & $(360)^{*}$ & 0.02 & $10^{b)}$ & 87 & 4 & $10^{e)}$ & 90 & 5 \\
\hline Pyrazophos & FPD & $(221)^{*}$ & 0.02 & $10^{b)}$ & 88 & 4 & $10^{e)}$ & 84 & 15 \\
\hline Metolcarb & EI & 108 & 0.02 & $10^{b)}$ & 82 & 11 & $25^{d)}$ & 81 & 29 \\
\hline Isoprocarb & EI & 136 & 0.02 & $10^{b)}$ & 89 & 12 & $25^{d)}$ & 91 & 15 \\
\hline $\mathrm{XMC}$ & EI & 122 & 0.02 & $10^{b)}$ & 80 & 12 & $25^{d)}$ & 87 & 20 \\
\hline Xylylcarb & EI & 122 & 0.02 & $10^{b)}$ & 86 & 10 & $19^{e)}$ & 83 & 19 \\
\hline Tecnazene & EI & 203 & 0.01 & $10^{b)}$ & 77 & 9 & $10^{g)}$ & 82 & 8 \\
\hline Fenobucarb & EI & 121 & 0.02 & $10^{b)}$ & 87 & 8 & $25^{d)}$ & 89 & 14 \\
\hline Propachlor & EI & 120 & 0.02 & $10^{b)}$ & 87 & 8 & $10^{g)}$ & 83 & 5 \\
\hline Propoxur & EI & 110 & 0.02 & $10^{b)}$ & 86 & 9 & $25^{d)}$ & 83 & 19 \\
\hline Chlorpropham & EI & 127 & 0.02 & $10^{b)}$ & 87 & 13 & $25^{d)}$ & 90 & 12 \\
\hline Bendiocarb & EI & 151 & 0.03 & $10^{b)}$ & 82 & 20 & $20^{e)}$ & 79 & 30 \\
\hline
\end{tabular}


Table 2. (Continued)

\begin{tabular}{|c|c|c|c|c|c|c|c|c|c|}
\hline Spiking level $(\mu \mathrm{g} / \mathrm{g})$ & & & & & 0.05 & & & 0.1 & \\
\hline Compound & Detector & $\begin{array}{l}\text { Monitor ion } \\
\qquad(m / z)\end{array}$ & $\begin{array}{l}\text { LOD } \\
(\mu \mathrm{g} / \mathrm{g})\end{array}$ & $\mathrm{n}$ & $\begin{array}{c}\text { Mean } \\
\text { recovery }(\%)\end{array}$ & $\begin{array}{l}\text { RSD } \\
(\%)\end{array}$ & $\mathrm{n}$ & $\begin{array}{c}\text { Mean } \\
\text { recovery }(\%)\end{array}$ & $\begin{array}{l}\text { RSD } \\
(\%)\end{array}$ \\
\hline Trifluralin & EI & 264 & 0.02 & $10^{b)}$ & 86 & 10 & $25^{d)}$ & 85 & 13 \\
\hline Benfluralin & EI & 292 & 0.01 & $10^{b)}$ & 86 & 7 & $10^{g)}$ & 84 & 10 \\
\hline Dicloran & EI & 176 & 0.01 & $10^{b)}$ & 81 & 6 & $10^{g)}$ & 83 & 11 \\
\hline Simazine & EI & 201 & 0.02 & $10^{b)}$ & 86 & 9 & $10^{g)}$ & 87 & 8 \\
\hline Carbofuran & EI & 164 & 0.03 & $10^{b)}$ & 84 & 17 & $20^{e)}$ & 89 & 24 \\
\hline Atrazine & EI & 200 & 0.02 & $10^{b)}$ & 88 & 9 & $10^{g)}$ & 85 & 8 \\
\hline Clomazone & EI & 204 & 0.02 & $10^{b)}$ & 85 & 10 & $10^{g)}$ & 84 & 6 \\
\hline Quintozene & EI & 237 & 0.02 & $10^{b)}$ & 88 & 9 & $10^{g)}$ & 82 & 9 \\
\hline Propyzamide & EI & 173 & 0.02 & $10^{b)}$ & 88 & 9 & $10^{g)}$ & 85 & 7 \\
\hline Pyrimethanil & EI & 198 & 0.02 & $10^{b)}$ & 85 & 10 & $10^{g)}$ & 83 & 6 \\
\hline Tri-allat & EI & 268 & 0.02 & $10^{b)}$ & 79 & 14 & $10^{g)}$ & 77 & 8 \\
\hline Benoxacor & EI & 120 & 0.02 & $10^{b)}$ & 91 & 9 & $10^{g)}$ & 88 & 8 \\
\hline Pirimicarb & EI & 166 & 0.01 & $10^{b)}$ & 93 & 5 & $25^{d)}$ & 88 & 10 \\
\hline Ethiofencarb & EI & 168 & 0.02 & $10^{b)}$ & 78 & 17 & $20^{e)}$ & 67 & 24 \\
\hline Benfuresate & EI & 163 & 0.01 & $10^{b)}$ & 87 & 6 & $25^{d)}$ & 87 & 8 \\
\hline Propanil & EI & 161 & 0.02 & $10^{b)}$ & 83 & 9 & $10^{g)}$ & 82 & 6 \\
\hline Bromobutide & EI & 232 & 0.02 & $10^{b)}$ & 86 & 9 & $10^{g)}$ & 83 & 7 \\
\hline Dimethenamid & EI & 154 & 0.01 & $10^{b)}$ & 88 & 7 & $25^{d)}$ & 89 & 12 \\
\hline Metribuzin & EI & 198 & 0.02 & $10^{b)}$ & 82 & 12 & $25^{d)}$ & 80 & 16 \\
\hline Acetochlor & EI & 223 & 0.01 & $10^{b)}$ & 87 & 7 & $10^{g)}$ & 81 & 8 \\
\hline Vinclozolin & EI & 285 & 0.02 & $10^{b)}$ & 94 & 9 & $10^{g)}$ & 82 & 6 \\
\hline Simetryn & EI & 213 & 0.02 & $10^{b)}$ & 85 & 9 & $10^{g)}$ & 84 & 8 \\
\hline Carbaryl & EI & 144 & 0.04 & $10^{b)}$ & 82 & 26 & $20^{e)}$ & 69 & 50 \\
\hline Alachlor & EI & 188 & 0.02 & $10^{b)}$ & 89 & 12 & $25^{d)}$ & 88 & 12 \\
\hline Ametryn & EI & 227 & 0.03 & $10^{b)}$ & 75 & 25 & $10^{g)}$ & 58 & 44 \\
\hline Prometryn & EI & 241 & 0.02 & $10^{b)}$ & 87 & 8 & $10^{g)}$ & 85 & 4 \\
\hline Metalaxyl & EI & 160 & 0.02 & $10^{b)}$ & 92 & 9 & $10^{g)}$ & 84 & 12 \\
\hline Ethofumesate & EI & 207 & 0.02 & $10^{b)}$ & 89 & 12 & $10^{g)}$ & 80 & 14 \\
\hline Esprocarb & EI & 222 & 0.02 & $10^{b)}$ & 81 & 14 & $25^{d)}$ & 83 & 13 \\
\hline Bromacil & EI & 205 & 0.04 & $10^{b)}$ & 77 & 31 & $10^{g)}$ & 85 & 21 \\
\hline Probenazole & EI & 130 & 0.03 & $9^{b)}$ & 57 & 26 & $10^{g)}$ & 71 & 26 \\
\hline Thiobencarb & EI & 257 & 0.03 & $10^{b)}$ & 75 & 19 & $10^{g)}$ & 82 & 17 \\
\hline Diethofencarb & EI & 225 & 0.04 & $10^{b)}$ & 76 & 34 & $25^{d)}$ & 84 & 18 \\
\hline Metolachlor & EI & 162 & 0.02 & $10^{b)}$ & 84 & 9 & $25^{d)}$ & 86 & 8 \\
\hline Fenpropimorf & EI & 128 & 0.02 & $10^{b)}$ & 87 & 10 & $10^{g)}$ & 82 & 7 \\
\hline Cyanazine & EI & 225 & 0.02 & $10^{b)}$ & 79 & 15 & $10^{g)}$ & 68 & 41 \\
\hline Triadimefon & EI & 208 & 0.02 & $10^{b)}$ & 94 & 9 & $25^{d)}$ & 82 & 11 \\
\hline Chlorthal-dimethyl & EI & 301 & 0.02 & $10^{b)}$ & 87 & 9 & $10^{g)}$ & 81 & 3 \\
\hline Nitrothal-isopropyl & EI & 236 & 0.02 & $10^{b)}$ & 80 & 9 & $10^{g)}$ & 77 & 8 \\
\hline Tetraconazole & EI & 336 & 0.02 & $10^{b)}$ & 89 & 10 & $20^{e)}$ & 84 & 15 \\
\hline Fthalide & EI & 243 & 0.02 & $10^{b)}$ & 86 & 11 & $10^{g)}$ & 80 & 12 \\
\hline
\end{tabular}


Table 2. (Continued)

\begin{tabular}{|c|c|c|c|c|c|c|c|c|c|}
\hline Spiking level $(\mu \mathrm{g} / \mathrm{g})$ & & & & & 0.05 & & & 0.1 & \\
\hline Compound & Detector & $\begin{array}{l}\text { Monitor ion } \\
\qquad(\mathrm{m} / \mathrm{z})\end{array}$ & $\begin{array}{l}\text { LOD } \\
(\mu \mathrm{g} / \mathrm{g})\end{array}$ & $\mathrm{n}$ & $\begin{array}{c}\text { Mean } \\
\text { recovery }(\%)\end{array}$ & $\begin{array}{l}\text { RSD } \\
(\%)\end{array}$ & $\mathrm{n}$ & $\begin{array}{c}\text { Mean } \\
\text { recovery }(\%)\end{array}$ & $\begin{array}{l}\text { RSD } \\
(\%)\end{array}$ \\
\hline Diphenamid & EI & 167 & 0.02 & $10^{b)}$ & 86 & 11 & $10^{g)}$ & 85 & 15 \\
\hline Dimethametryn & EI & 212 & 0.02 & $10^{b)}$ & 91 & 9 & $10^{g)}$ & 85 & 10 \\
\hline Pendimethalin & EI & 252 & 0.02 & $10^{b)}$ & 83 & 10 & $25^{d)}$ & 84 & 6 \\
\hline Penconazole & EI & 248 & 0.02 & $10^{b)}$ & 87 & 11 & $25^{d)}$ & 88 & 10 \\
\hline Pyrifenox & EI & 262 & 0.02 & $10^{b)}$ & 85 & 11 & $25^{d)}$ & 75 & 22 \\
\hline Triadimenol & EI & 168 & 0.03 & $5^{c)}$ & 83 & 23 & $20^{e)}$ & 93 & 52 \\
\hline Triflumizole & EI & 218 & 0.03 & $10^{b)}$ & 87 & 22 & $10^{g)}$ & IF & - \\
\hline Chinomethionate & EI & 206 & 0.02 & $10^{b)}$ & 26 & 45 & $20^{e)}$ & 38 & 48 \\
\hline Pacrobutrazol & EI & 236 & 0.04 & $10^{b)}$ & 74 & 27 & $25^{d)}$ & 87 & 17 \\
\hline$\alpha$-Endosulfan & EI & 241 & 0.03 & $5^{c)}$ & 93 & 18 & $10^{g)}$ & IF & - \\
\hline Butachlor & EI & 160 & 0.02 & $10^{b)}$ & 90 & 11 & $5^{l)}$ & 72 & 12 \\
\hline Flutriafol & EI & 123 & 0.02 & $5^{c)}$ & 88 & 13 & $10^{g)}$ & IF & - \\
\hline Napropamide & EI & 128 & 0.02 & $10^{b)}$ & 95 & 13 & $10^{g)}$ & IF & - \\
\hline Flutolanil & EI & 173 & 0.02 & $10^{b)}$ & 92 & 10 & $25^{d)}$ & 89 & 21 \\
\hline Hexaconazole & EI & 214 & 0.02 & $10^{b)}$ & 88 & 13 & $20^{e)}$ & 88 & 20 \\
\hline Isoprothiolane & EI & 204 & 0.03 & $10^{b)}$ & 81 & 19 & $10^{g)}$ & 89 & 12 \\
\hline Metominostrobin E & EI & 191 & 0.02 & $10^{b)}$ & 95 & 13 & $10^{g)}$ & 86 & 14 \\
\hline Uniconazole & EI & 234 & 0.02 & $10^{b)}$ & 95 & 10 & $20^{f)}$ & 91 & 18 \\
\hline Pretilachlor & EI & 238 & 0.02 & $10^{b)}$ & 94 & 9 & $25^{d)}$ & 90 & 11 \\
\hline Fludioxonil & EI & 248 & 0.02 & $10^{b)}$ & 84 & 8 & $25^{d)}$ & 85 & 13 \\
\hline Oxadiazon & EI & 175 & 0.02 & $10^{b)}$ & 84 & 12 & $10^{g)}$ & 81 & 8 \\
\hline Flamprop-methyl & EI & 230 & 0.01 & $10^{b)}$ & 93 & 7 & $10^{g)}$ & 85 & 7 \\
\hline Myclobutanil & EI & 179 & 0.02 & $10^{b)}$ & 88 & 12 & $25^{d)}$ & 78 & 28 \\
\hline Oxyfluorfen & EI & 252 & 0.02 & $10^{b)}$ & 87 & 11 & $10^{g)}$ & 86 & 8 \\
\hline Buprofezin & EI & 175 & 0.02 & $10^{b)}$ & 91 & 14 & $10^{g)}$ & 80 & 33 \\
\hline Flusilazole & EI & 233 & 0.02 & $10^{b)}$ & 90 & 11 & $25^{d)}$ & 82 & 14 \\
\hline Bupirimate & EI & 193 & 0.02 & $10^{b)}$ & 93 & 10 & $10^{g)}$ & 86 & 10 \\
\hline Kresoxim-methyl & EI & 116 & 0.02 & $10^{b)}$ & 92 & 9 & $25^{d)}$ & 88 & 14 \\
\hline Metominostrobin Z & EI & 191 & 0.02 & $10^{b)}$ & 90 & 14 & $10^{g)}$ & 81 & 10 \\
\hline Cyproconazole & EI & 222 & 0.02 & $10^{b)}$ & 87 & 12 & $20^{e)}$ & 88 & 13 \\
\hline$\beta$-Endosulfan & EI & 241 & 0.04 & $10^{b)}$ & 88 & 29 & $5^{h)}$ & 56 & 24 \\
\hline Oxadixyl & EI & 132 & 0.03 & $10^{b)}$ & 87 & 19 & $10^{g)}$ & 94 & 12 \\
\hline Mepronil & EI & 269 & 0.04 & $10^{b)}$ & 92 & 24 & $25^{d)}$ & 80 & 24 \\
\hline Fluacrypyrim & EI & 204 & 0.02 & $10^{b)}$ & 93 & 9 & $10^{g)}$ & 81 & 6 \\
\hline Carfentrazone-ethyl & EI & 312 & 0.02 & $10^{b)}$ & 94 & 11 & $10^{g)}$ & 89 & 16 \\
\hline Diofenolan & EI & 186 & 0.02 & $10^{b)}$ & 92 & 13 & $10^{g)}$ & 87 & 17 \\
\hline Benalaxyl & EI & 148 & 0.02 & $10^{b)}$ & 90 & 8 & $10^{g)}$ & 87 & 12 \\
\hline Quinoxyfen & EI & 237 & 0.02 & $10^{b)}$ & 84 & 9 & $10^{g)}$ & 83 & 10 \\
\hline Norflurazon & EI & 303 & 0.02 & $10^{b)}$ & 93 & 13 & $10^{g)}$ & 87 & 9 \\
\hline Lenacil & EI & 153 & 0.02 & $10^{b)}$ & 91 & 9 & $25^{d)}$ & 84 & 17 \\
\hline Trifloxystrobin & EI & 116 & 0.03 & $10^{b)}$ & 93 & 15 & $10^{g)}$ & 85 & 11 \\
\hline
\end{tabular}


Table 2. (Continued)

\begin{tabular}{|c|c|c|c|c|c|c|c|c|c|}
\hline Spiking level $(\mu \mathrm{g} / \mathrm{g})$ & & & & & 0.05 & & & 0.1 & \\
\hline Compound & Detector & $\begin{array}{c}\text { Monitor ion } \\
(\mathrm{m} / \mathrm{z})\end{array}$ & $\begin{array}{l}\text { LOD } \\
(\mu \mathrm{g} / \mathrm{g})\end{array}$ & $\mathrm{n}$ & $\begin{array}{c}\text { Mean } \\
\text { recovery }(\%)\end{array}$ & $\begin{array}{l}\text { RSD } \\
(\%)\end{array}$ & $\mathrm{n}$ & $\begin{array}{c}\text { Mean } \\
\text { recovery }(\%)\end{array}$ & $\begin{array}{l}\text { RSD } \\
(\%)\end{array}$ \\
\hline Hexazinone & EI & 171 & 0.02 & $10^{b)}$ & 85 & 11 & $10^{g)}$ & 75 & 20 \\
\hline Tebuconazole & EI & 250 & 0.02 & $10^{b)}$ & 86 & 12 & $25^{d)}$ & 86 & 17 \\
\hline Diclofop-methyl & EI & 340 & 0.02 & $10^{b)}$ & 96 & 9 & $10^{g)}$ & 88 & 16 \\
\hline Thenylchlor & EI & 288 & 0.02 & $10^{b)}$ & 87 & 15 & $25^{d)}$ & 87 & 15 \\
\hline Propargite & EI & 135 & 0.02 & $5^{c)}$ & 85 & 12 & $5^{h)}$ & 82 & 48 \\
\hline Diflufenican & EI & 266 & 0.02 & $10^{b)}$ & 89 & 13 & $25^{d)}$ & 87 & 12 \\
\hline Pyributicarb & EI & 165 & 0.02 & $10^{b)}$ & 90 & 13 & $10^{g)}$ & 84 & 21 \\
\hline Iprodione & EI & 314 & 0.05 & $8^{b)}$ & 90 & 32 & $19^{e)}$ & 82 & 23 \\
\hline Brompropylate & EI & 341 & 0.04 & $9^{b)}$ & 102 & 25 & $10^{g)}$ & IF & - \\
\hline Bifenthrin & EI & 181 & 0.02 & $10^{b)}$ & 88 & 9 & $25^{d)}$ & 87 & 10 \\
\hline Picolinafen & EI & 376 & 0.02 & $10^{b)}$ & 93 & 11 & $10^{g)}$ & 92 & 11 \\
\hline Methoxychlor & EI & 227 & 0.02 & $10^{b)}$ & 91 & 10 & $10^{g)}$ & 86 & 14 \\
\hline Fenpropathrin & EI & 265 & 0.02 & $5^{c)}$ & 79 & 9 & $20^{e)}$ & 96 & 10 \\
\hline Tebufenpyrad & EI & 333 & 0.04 & $10^{b)}$ & 59 & 45 & $25^{d)}$ & 91 & 13 \\
\hline Phenothrin & EI & 183 & 0.02 & $10^{b)}$ & 85 & 15 & $10^{g)}$ & 87 & 32 \\
\hline Tetradifon & EI & 356 & 0.03 & $5^{c)}$ & 118 & 39 & $5^{h)}$ & 88 & 10 \\
\hline Furametpyr & EI & 298 & 0.02 & $10^{b)}$ & 93 & 12 & $25^{d)}$ & 88 & 17 \\
\hline Pyriproxyfen & EI & 136 & 0.03 & $10^{b)}$ & 96 & 16 & $10^{g)}$ & 83 & 26 \\
\hline Cyhalofop-butyl & EI & 357 & 0.03 & $10^{b)}$ & 105 & 32 & $10^{g)}$ & 87 & 9 \\
\hline Mefenacet & EI & 192 & 0.03 & $10^{b)}$ & 86 & 22 & $24^{d)}$ & 94 & 24 \\
\hline Fenarimol & EI & 139 & 0.02 & $10^{b)}$ & 90 & 8 & $24^{d)}$ & 85 & 24 \\
\hline Bitertanol & EI & 170 & 0.03 & $10^{b)}$ & 90 & 18 & $25^{d)}$ & 87 & 35 \\
\hline Pyridaben & EI & 147 & 0.04 & $10^{b)}$ & 73 & 28 & $10^{g)}$ & IF & - \\
\hline Fenbuconazole & EI & 129 & 0.04 & $9^{b)}$ & 99 & 26 & $10^{g)}$ & IF & - \\
\hline Pyrimidifen & EI & 184 & 0.03 & $5^{b)}$ & 65 & 26 & $24^{d)}$ & 89 & 16 \\
\hline
\end{tabular}

${ }^{a)}$ Recovery data for orange, paprika, lettuce and tomato. ${ }^{b)}$ Recovery data for lettuce and tomato. ${ }^{c)}$ Recovery data for tomato. ${ }^{d)}$ Recovery data for apple, banana, grapefruit, broccoli and spinach. ${ }^{e}$ Recovery data for apple, banana, grapefruit and broccoli. ${ }^{f)}$ Recovery data for apple, banana, grapefruit and spinach. ${ }^{g)}$ Recovery data for broccoli and spinach. ${ }^{h)}$ Recovery data for broccoli. ${ }^{i)}$ Recovery data for spinach. ND: Not detected. IF: Interfered.

* Also monitored by EI mode GC/MS.

identification. A few organophosphorous pesticides were measured with GC/MS because of interference in broccoli. Captan was not recovered from spinach and broccoli. Captafol was not recovered from spinach, it was poorly recovered in broccoli, and its recovery was fractured in other crops. Carbaryl, endosulfan, pyridaben, fenbuconazole and probenazole showed low recoveries and/or high RSDs in tested crops. The method was considered a screening procedure for these compounds. The limits of detection (LODs) were defined as 3 times the standard deviation of 5-25 replicate analyses of samples fortified at 0.05 or $0.1 \mu \mathrm{g} / \mathrm{g}$ with EI mode GC/MS. The LODs of the pesticides detected with GC/FPD and NCI mode GC/MS were calculated based on the noise levels on the chromatograms of the blank sample solution and the respective standard peaks, since serious interfering peaks were not observed. In this work, the minimum LOD was defined as $0.01 \mu \mathrm{g} / \mathrm{g}$ to take account of instrumental dispersion.

The proposed method shows good sensitivity and recovery and allows for rapid analysis. A single chemist can prepare 6 homogenized samples within $4 \mathrm{hr}$. The method requires only a small volume of solvent per sample and needs no special equipment. It covers a wide range of pesticides, is applicable to various fruits and vegetables, and is ideally suited for use in a regulatory laboratory. Further research will focus on the expansion of this method to other pesticides. 


\section{REFERENCES}

1) J. Fillion, R. Hindle, M. Lacroix and J. Selwyn: J. AOAC Int. $\mathbf{7 8}$, 1252-1266 (1995).

2) T. Nagayama, M. Kobayashi, H. Shioda and Y. Tamura: J. Food Hyg. Soc. Japan 36, 643-655 (1995) (in Japanese).

3) Y. Akiyama, M. Yano, T. Mitsuhashi, N. Takeda and M. Tauji: $J$. Food Hyg. Soc. Japan 37, 351-362 (1996) (in Japanese).

4) J. Fillion, F. Sauve and J. Selwyn: J. AOAC Int. 83, 698-713 (2000).

5) S. Nemoto, K. Sasaki, S. Eto, I. Saito, H. Sakai, T. Takahashi, Y. Tonogai, T. Nagayama, S. Hori, Y. Maekawa and M. Toyoda: $J$. Food Hyg. Soc. Japan 41, 233-241 (2000) (in Japanese).

6) H. Obana, K. Akutsu, M. Okihashi and S. Hori: Analyst 126, 1529-1534 (2001).

7) A. Kaihara, K. Yoshii, Y. Tsumura, Y. Nakamura, S. Ishimitsu and Y. Tonogai: J. Health Sci. 46, 336-342 (2000).

8) J. W. Pensabene, W. Fiddler and D. J. Donoghue: J. Agric. Food Chem. 48, 1668-1672 (2000).

9) B. E. Richter, B. A. Jones, J. L. Ezzell, N. L. Porter, N. Av- dalovic and C. Pohl: Anal. Chem. 68, 1033-1039 (1996).

10) H. Obana, K. Kikuchi, M. Okihashi and S. Hori: Analyst 122, 217-220 (1997).

11) M. Okihashi, H. Obana and S. Hori: Analyst 123, 711-714 (1998).

12) M. Okihashi, H. Obana and S. Hori: J. Food Hyg. Soc. Japan 38, 16-21 (1997) (in Japanese).

13) E. Ueno, H. Oshima, I. Saito and H. Matsumoto: J. AOAC Int. 87, 1003-1015 (2004).

14) M. Anastassiades, S. J. Lehotay, D. Stajnbaher and F. J. Schenck: J. AOAC Int. 86, 412-431 (2003).

15) F. J. Schenck and J. E. Hobbs: Bull. Environ. Contam. Toxicol. 73, 24-30 (2004).

16) H. Obana, M. Okihashi, K. Akutsu, S. Taguchi and S. Hori: $J$. Food Hyg. Soc. Japan 42, 148-153 (2001) (in Japanese).

17) H. Obana, S. Hori, M. Okihashi and T. Nishimune: Jpn. J. Food Chem. 1, 2-7 (1994).

18) Y. Saito, S. Kodama, A. Matsunaga and A. Yamamoto: J. $A O A C$ Int. 87, 1356-1367 (2004). 